\title{
Rick Morimoto
}

\section{A pioneer in proteostasis is changing the way we think about organismal biology and human disease.}

$\mathrm{R}$ ick Morimoto recalls his fascination with a seminar given by Harvard University professor Matt Meselson that would change the course of his scientific career: "Who would have imagined that a fruit fly would have a thermometer? And what does a thermometer look like in a fruit fly?" Meselson's description of the heat shock response in Drosophila resonated with Morimoto, then a graduate student at the University of Chicago, in reaching from the molecular details of a biological process to an outcome at the organismal level. His continued appreciation for science at different length scales has played a critical part in a quiet revolution in scientific thinking: the conceptualizing of 'proteostasis' as a new framework for understanding cellular, tissue and organismal function.

As a professor at Northwestern University, Morimoto established his own laboratory focused on the heat shock transcription factor and molecular chaperones. He remembers that "when the heat shock response field was founded, people working on the proteasome, ubiquitin-mediated degradation and translation were all together because there wasn't much data, but there was a sense that these were all related." Yet as the fields matured, they diverged, such that scientists attended dedicated chaperone meetings or stress response conferences, and cross-talk between fields stagnated. 'Proteostasis', defined in a seminal review by William Balch, Morimoto, Andrew Dillin and Jeffery Kelly (Science 319, 916-919, 2008), was a reflection of the authors' discovery that, according to Morimoto, "to understand the complexity, we had to now cross over and not just think about each response, each entity alone."

Proteostasis, though formally a contraction of protein homeostasis, encompasses much larger themes of convergence and interdependence among topics such as protein synthesis, folding, transport and degradation. The term is not meant to group related researchers together but rather to recognize that the systems themselves are integrated. As Morimoto explains, "if you change the activity of the proteasome-alter its selectivity-what else has adjusted? That's the beauty of the system." The concept includes intracellular

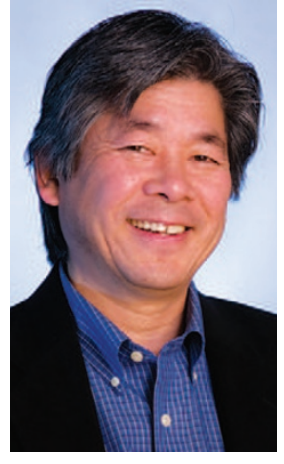

"If you
change the
activity of the
proteasome...
what else has
adjusted?
That's the
beauty of the
system."

as well as intercellular networks of systems that respond to and can compensate for each other. Morimoto describes, "it was a recognition that understanding how each of the parts is moving simultaneously in each setting helps us go from deep fundamentals on the processes of amyloid formation and on the intrinsic properties of sequences all the way up to organismal biology."

Researchers across scientific disciplines have embraced the proteostasis framework, initiating new conference series and research institutes. Elke Deuerling is Chair of Molecular Microbiology and Spokesperson of the new Collaborative Research Center 969, 'Chemical and Biological Principles of Cellular Proteostasis', located at the University of Konstanz (http://www.sfb969. uni-konstanz.de/home/). Deuerling says the center reflects a "common desire to better understand the intricate network and underlying chemical and biological processes that contribute to proteostasis." Mark Wilson, a founding member of the University of Wollongong's Proteostasis and Disease Research Centre (PDRC; http://smah.uow. edu.au/pdrc/index.html), describes the central inspiration for founding the PDRC as more of a "growing realization that all our individual research directions, and more, were encompassed by the concept of proteostasis."

In changing the way we describe human biology, proteostasis also has implications for how we treat deviations from optimal homeostasis. Indeed, Wilson believes that the field has "raised the possibility that a process as fundamental as aging itself may result from dysfunctions in proteostasis." Morimoto agrees, suggesting that proteostasis provides "a very unique opportunity to identify small molecules that can retune the system when it changes, restore it during aging and perhaps even audaciously restore a damaged tissue that's been expressing a misfolded, aggregation-prone protein for a long period."

Of course, this retuning could be important in more egregious dysfunctions as well, such as neurodegenerative disease. Morimoto argues that successful drug discovery efforts will depend on consolidating validated disease models and testing hit compounds across model systems both to gain confidence in how a compound affects one disease and to learn more about how similar mechanisms might affect multiple diseases in similar or disparate ways. He envisions that a shared platform of some kind could be transformative, noting "The field's exactly at that moment where we've got the coolest set of results, but it's still individual sets of results and individual laboratories without much ability to cross-correlate."

Work from Morimoto's laboratory looking at these 'retuning mechanisms' in Caenorhabditis elegans has also led to what Deuerling describes as one of the most surprising findings related to proteostasis thus far: cell-nonautonomous stress responses in multicellular organisms. As Deuerling explains, this is "where neuronal cells sense and transmit signals to non-neuronal somatic tissues to instruct these cells with the appropriate stress responses that induce chaperones and other quality control systems in order to prevent or combat proteome damage throughout the organisms." Balch, at the Scripps Research Institute, puts it more plainly: "How your stubbed toe knows to be repaired is managed by your brain in completely unanticipated ways."

The full implications of this finding are still unclear, but Morimoto is eager to learn more. He hypothesizes, "If stress responses can be activated cell non-autonomously, because of intrinsic connections of tissues, could one use that strategy to restore a misfolded protein or restore a tissue without directly targeting that tissue?" Balch believes "the proteostasis paradigm shift is going to take over medicine as it is practiced today, from neurodegeneration to cancer. We just need to learn the rules."

\section{Catherine Goodman Cambridge, Massachusetts}

Corrected after print 11 December 2014 


\section{ERRATUM}

\section{Rick Morimoto}

Catherine Goodman

Nat. Chem. Biol. 10, 875 (2014); published online 17 October 2014; corrected after print 11 December 2014

In the version of this article initially published, Mark Wilson was inadvertently described as 'the' founding member of the PDRC, instead of 'a' founding member. The error has been corrected in the HTML and PDF versions of the article. 